gr-qc/9402034, CGPG-94/2-2

\title{
Is the exponential of the Chern-Simons action a normalizable physical state?
}

\author{
Guillermo A. Mena Marugán \\ Center for Gravitational Physics and Geometry, \\ Pennsylvania State University, 104 Davey Laboratory, \\ University Park, PA 16802, USA. \\ On leave from: Instituto de Matemáticas y Física Fundamental, \\ C.S.I.C., Serrano 121, 28006 Madrid, Spain.
}

February, 1994

\begin{abstract}
We determine the wavefunction that corresponds to the exponential of the ChernSimons action in a family of gravitational minisuperspace models provided with cosmological constant whose non-perturbative canonical quantization is completely known. We show that this wavefunction does not represent a proper quantum state, because it is not normalizable with respect to the unique inner product of Lorentzian gravity.
\end{abstract}

PACS number: 04.60.+n 
The recent formulation by Ashtekar [1,2] of an alternative formalism for the description of General Relativity has renewed the hopes of constructing a consistent quantum theory of gravity by implementing Dirac's canonical quantization program [3]. In addition to a change of emphasis from geometrodynamics to connection dynamics, Ashtekar has introduced a proposal to determine the inner product in the space of solutions to all quantum gravitational constraints (the space of physical states). One must find first a sufficient large number of gravitational observables, that is, functions on phase space that commute with all the constraints. The inner product is then uniquely fixed [4] by demanding that those observables that correspond to real classical variables are represented in the quantum theory by self-adjoint operators. These self-adjointness requirements are usually called reality conditions [2].

Among the different achievements reached so far in the Ashtekar formalism, one of the more significant successes has been the discovery, for the first time, of an exact solution to the quantum constraints of full General Relativity. This physical state is provided by the exponential of the Chern-Simons action [5], and its explicit form has been obtained both in the connection $[6,7]$ and in the loop representation [5] of quantum gravity constructed using Ashtekar variables $[2,8]$. Since this solution (the Chern-Simons solution, from now on) is the only exact state that is known for full gravity with a non-vanishing cosmological term, it would be extremely interesting to determine whether it belongs to the Hilbert space of physical states for Lorentzian General Relativity, i.e., if it actually represents a quantum gravitational state of finite norm. In the present circumstances, however, it seems impossible to arrive at a definitive conclusion about the normalizability of the Chern-Simons solution, because the expression of the inner product for Lorentzian gravity has not been found yet. A partial analysis can none the less be carried out by restricting our discussion to gravitational minisuperspace models provided with a cosmological constant for 
which both the physical inner product and the Chern-Simons wavefunction can be obtained.

A lot of attention $[6,9]$ has been devoted lately to the quantization of minisuperspace models as a powerful tool to check the consistency of the quantization program proposed by Ashtekar, as well as to develop the mathematical machinery that will be presumably needed to quantize the full theory of gravity. There exist several examples in the literature in which the non-perturbative canonical quantization has been performed to completion, including the determination of the inner product $[10,11]$. In all these examples, nevertheless, the cosmological constant has been assumed to vanish, except (to our knowledge) in the canonical quantization of a family of anisotropic models that contains the locally rotationally symmetric (LRS) Bianchi types I and III and the Kantowski-Sachs model as particular cases $[11,12]$. Our aim in this letter is to find the explicit form of the Chern-Simons solution in this family of minisuperspaces and show that, with the inner product that corresponds to Lorentzian gravity, such a wavefunction possesses an infinite norm, so that it cannot be considered a proper quantum state.

The gravitational models that we are going to analyse can be described by the spacetime metric [12]:

$$
d s^{2}=-\frac{\bar{N}^{2}(t)}{a^{2}(t)} d t^{2}+a^{2}(t) d r^{2}+b^{2}(t) d \Omega_{2}^{2} .
$$

Here, the coordinate $r$ is periodic with period equal to $2 \pi, a$ and $b$ are the two scale factors of the model, $\bar{N}$ is the rescaled lapse function, and $d \Omega_{2}^{2}$ denotes the metric of a compact orientable two-manifold with constant scalar of curvature equal to $2 k$, $k=+1,0$ or -1 , and volume given by $V_{2}$. The two-metric $d \Omega_{2}^{2}$ can always be written locally as

$$
d \Omega_{2}^{2}=d \alpha^{2}+f^{2}(\alpha) d \beta^{2}
$$


where $f(\alpha)$ satisfies the differential equation

$$
\partial_{\alpha}^{2} f(\alpha)=-k f(\alpha)
$$

The specific minisuperspace considered depends thus on the value taken by $k$. For $k=+1$ we get the Kantowski-Sachs model, while for $k=0$ and -1 we obtain, respectively, the LRS Bianchi types I and III [12].

From now on, we will adopt the system of units in which $\hbar=1,8 \pi G=1$, and $4 \pi V_{2}=1, G$ being the gravitational constant. The Hamiltonian constraint of the minisuperspaces (1), including the contribution of the cosmological constant $\lambda$, can then be expressed in the form $[11,12]$ :

$$
\mathcal{H}=\frac{1}{2}\left(-4 p_{c} p_{b}+\lambda b^{2}-k\right)=0,
$$

with $p_{b}$ and $p_{c}$ the momenta canonically conjugate to $b$ and

$$
c=a^{2} b
$$

The quantization of these models was first analysed by Halliwell and Louko by using the complex path-integral approach in the geometrodynamic formulation [12]. The complete non-perturbative canonical quantization of these systems was presented in Ref. [11], where the unique inner product compatible with the reality conditions of Lorentzian gravity was determined.

Since we are interested in studying the behaviour of the Chern-Simons wavefunction, which is originally defined in the connection representation of the Ashtekar formulation [5-7], it will be useful to begin our discussion by introducing the Ashtekar variables for the models under consideration. Once we have found the explicit form of the Chern-Simons solution, we will translate our results into the representation that was used in [11] to achieve the canonical quantization, i.e., the representation in which we know the physical inner product. 
The Ashtekar variables are a densitized triad, $\tilde{E}_{i}^{a}$, and a conjugate $\mathrm{SO}(3)$ connection, $A_{a}^{i}[1,2]$. The lower case Latin letters from the beginning and the middle of the alphabet denote here spatial and $\mathrm{SO}(3)$ indices respectively, the latter being raised and lowered with the metric $\eta^{i j}=(1,1,1)$. For non-degenerate metrics, the Ashtekar variables can be obtained from the inverse triad, $e_{i}^{a}$, and the extrinsic curvature, $K_{a b}$,

$$
\tilde{E}_{i}^{a}=e_{i}^{a} q(e), \quad A_{a}^{i}=\Gamma_{a}^{i}(e)-i K_{a b} e_{i}^{b}
$$

$q(e)$ being the determinant of the triad and $\Gamma_{a}^{i}(e)$ the $\mathrm{SO}(3)$ connection compatible with the three-metric [2]:

$$
\Gamma_{a}^{i}(e)=-\frac{1}{2} \epsilon^{i j k} \underset{\sim}{E}{ }_{j b}\left(\partial_{a} \tilde{E}_{k}^{b}+\Gamma_{c a}^{b} \tilde{E}_{k}^{c}\right)
$$

In this formula, $\epsilon^{i j k}$ denotes the antisymmetric symbol, $\Gamma_{b c}^{a}$ the Christoffel symbol [13], and $\underset{\sim}{E_{a}^{i}}$ the inverse of $\tilde{E}_{i}^{a}$.

Using the coordinatization of $(1,2)$ and imposing a convenient gauge condition on the $\mathrm{SO}(3)$ degrees of freedom (so that the densitized triad be diagonal), the particularization of these equations to our minisuperspace models leads to the expressions:

$$
\begin{gathered}
\tilde{E}_{1}^{1}=f(\alpha) x, \quad \tilde{E}_{2}^{2}=f(\alpha) y, \quad \tilde{E}_{3}^{3}=y \\
A_{1}^{1}=-2 i P_{x}, \quad A_{2}^{2}=-i P_{y}, \quad A_{3}^{3}=-i f(\alpha) P_{y} \\
A_{3}^{1}=\partial_{\alpha} f(\alpha),
\end{gathered}
$$

where we have introduced the new variables

$$
x=b^{2}, \quad y=a b,
$$

and $P_{x}$ and $P_{y}$ are the momenta canonically conjugate to $x$ and $y$. To obtain eq. (7) we have used the equations of motion derived from the Hamiltonian (4). The rest of components of the densitized triad and the Ashtekar connection vanish in the gauge selected here. 
From eqs. (6-8), it is clear that all the degrees of freedom of the Ashtekar variables reduce in this family of homogeneous models to the set of functions $x, y, P_{x}$ and $P_{y}$. On the other hand, only the Hamiltonian constraint remains to be imposed in these minisuperspaces, since we have already fixed the diffeomorphism and $\mathrm{SO}(3)$ gauge freedom. Substituting then eqs. (6-8) and eq. (3) in the general formula for Ashtekar's Hamiltonian constraint [2,14]:

$$
\begin{gathered}
\tilde{\mathcal{H}}=\frac{1}{2} \epsilon^{i j k} \eta_{a b c} \tilde{E}_{j}^{b} \tilde{E}_{k}^{c}\left(\tilde{\mathcal{F}}_{i}^{a}+\frac{\lambda}{3} \tilde{E}_{i}^{a}\right)=0 \\
\tilde{\mathcal{F}}_{i}^{a}=\tilde{\eta}^{a b c}\left(\partial_{b} A_{c i}+\frac{1}{2} \epsilon_{i j k} A_{b}^{j} A_{c}^{k}\right)
\end{gathered}
$$

(with $\tilde{\eta}^{a b c}$ the Levi-Civitta tensor-density) and integrating the result, divided by $f(\alpha)$ f, over each constant time surface, we arrive at a Hamiltonian of the form

$$
\overline{\mathcal{H}}=\frac{1}{2}\left(-4 x y P_{x} P_{y}-y^{2} P_{y}^{2}+\lambda x y^{2}-k y^{2}\right)=0
$$

For consistency in our calculations [2,14], this constraint must coincide with eq. (4) when multiplied by the time dependent part of the square root of the determinant of the three-metric (note that we have integrated over the spatial dependence to derive eq. (12)) and by a factor of $a$ that arises from the definition of the rescaled lapse function $\bar{N}$ in (1). That this is indeed the case can be checked by using eqs. (5) and (9) and the relations between the momenta $\left(P_{x}, P_{y}\right)$ and $\left(p_{b}, p_{c}\right)$ that follow from them:

$$
\frac{1}{y} P_{y}=\frac{2}{b} p_{c}, \quad P_{x}=\frac{1}{2 b} p_{b}-\frac{c}{2 b^{2}} p_{c} .
$$

The exponential of the Chern-Simons action is known to be a physical state in the connection representation of the Ashtekar formulation only if all the Ashtekar connections are gathered to the right of the densitized triads in the gravitational constraints before quantization $[5,6]$. Under reduction to our minisuperspace models, this prescription gives exactly the factor ordering that appears in eq. (12),

\footnotetext{
${ }^{*}$ Notice that $f(\alpha) \neq 0$ for non-degenerate metrics $(1,2)$.
} 
and the analogue of the connection representation is provided now by the $\left(P_{x}, P_{y}\right)$ representation, in which the operators $\hat{x}$ and $\hat{y}$ act as derivatives with respect to their conjugate momenta $\left(\hat{x}=i \partial_{P_{x}}\right.$ and $\left.\hat{y}=i \partial_{P_{y}}\right)$, and $\hat{P}_{x}$ and $\hat{P}_{y}$ are multiplicative operators.

The Chern-Simons wavefunction is in fact a quantum solution to a more restrictive equation than constraint (10), i.e.,

$$
\tilde{E}_{i}^{a}=-\frac{3}{\lambda} \tilde{\mathcal{F}}_{i}^{a}
$$

This condition, together with the factor ordering commented above, guarantees that the exponential of the Chern-Simons action is annihilated by the quantum Hamiltonian obtained from eq. (10). Moreover, when the topology of the constant time sections is fixed (like, for instance, in the models that we are studying), the ChernSimons solution is characterized, up to a constant, as the only wavefunction that satisfies the quantum version of relation (13) [7]. For the family of minisuperspaces analysed here, eqs. (3), (6-8) and (11) allow us to rewrite that relation as the following pair of identities:

$$
x=\frac{3}{\lambda}\left(k+P_{y}^{2}\right), \quad y=\frac{6}{\lambda} P_{x} P_{y}
$$

A straightforward calculation shows then that, in the $\left(P_{x}, P_{y}\right)$ representation, the only quantum solution to eqs. (14) is provided by

$$
\Psi_{\mathrm{CS}}\left(P_{x}, P_{y}\right)=\exp \left(-i \frac{3}{\lambda}\left[k P_{x}+P_{x} P_{y}^{2}\right]\right)
$$

which must be therefore the Chern-Simons wavefunction.

In order to transcribe these results into the representation in which the physical inner product is known [11], let us assume first that there exists a well-defined $(x, y)$ representation for the quantum theory with Hamiltonian constraint (12). In such a representation, $\hat{P}_{x}=-i \partial_{x}, \hat{P}_{y}=-i \partial_{y}$ and $\hat{x}$ and $\hat{y}$ act as multiplicative operators. Recalling eqs. (5) and (9), we can define then an equivalent $(b, c)$ representation 
through the following rescaling of the wavefunctions $\Psi(x, y)$,

$$
\Psi(b, c)=\frac{1}{\sqrt{b}} \Psi\left(x=b^{2}, y=\sqrt{c b}\right) .
$$

Applying now the chain rule in the quantum Hamiltonian constraint obtained from eq. (12), we conclude that

$$
\hat{\overline{\mathcal{H}}} \Psi(x, y)=\frac{c b \sqrt{b}}{2}\left(-4 \hat{p}_{b} \hat{p}_{c}+\lambda b^{2}-k\right) \Psi(b, c)=0,
$$

where $\hat{p}_{b}=-i \partial_{b}$ and $\hat{p}_{c}=-i \partial_{c}$. Therefore, if we neglect the prefactor $c b \sqrt{b}$ in the above equation, our previous assumption about the viability of a $(x, y)$ representation amounts to admit the existence of a $(b, c)$ representation for those models whose only quantum constraint is precisely the Wheeler-DeWitt equation that follows from eq. (4). That such a representation exists was proved in fact in Ref. [11], where we showed that, in the minisuperspaces with Hamiltonian $(4)$, the real $(b, c)$ representation is well-defined at least for all physical states in the Hilbert space of Lorentzian gravity. Thus, we can always adopt this $(b, c)$ representation to describe the quantum systems under consideration.

The global factor $c b \sqrt{b}$ that appears in (16) actually allows the wavefunctions $\Psi(b, c)$ to be Green functions of the Wheeler-DeWitt equation associated with eq. (4), rather than mere solutions. Eq. (16) is then satisfied in the distributional sense. We will return to this point later in this letter.

Let now $\Psi_{C S}(b, c)$ be the Chern-Simons wavefunction in the $(b, c)$ representation introduced above. We know that, in the models that we are discussing, this wavefunction must exist, at least, if the Chern-Simons solution is a normalizable physical state for Lorentzian gravity. A straightforward calculation employing eqs. (5), (9) and the chain rule shows then that the quantum version of eqs. (14), which determine the Chern-Simons wavefunction, translate in the $(b, c)$ representation into

$$
\sqrt{b}\left(k-\frac{\lambda}{3} b^{2}+\frac{4 c}{b} \hat{p}_{c}^{2}-\frac{2 i}{b} \hat{p}_{c}\right) \Psi_{C S}(b, c)=0,
$$




$$
\frac{\sqrt{c b}}{b^{2}} \sqrt{b}\left(\hat{p}_{b} \hat{p}_{c}-\frac{\lambda}{6} b^{2}-\frac{c}{b} \hat{p}_{c}^{2}+\frac{i}{2 b} \hat{p}_{c}\right) \Psi_{C S}(b, c)=0 .
$$

We are at last in an adequate position to rewrite our expressions in the representation that was used in Ref. [11] to achieve the non-perturbative canonical quantization of these systems. This quantization was obtained by means of a transformation to a new set of canonical variables, $(Q, P, H, T)$, related to the original set $\left(b, p_{b}, c, p_{c}\right)$ through the equations

$$
\begin{aligned}
& b=4 P T, \quad p_{b}=4 \lambda P T^{2}-\frac{1}{4 P} H, \\
& c=Q+\frac{16}{3} \lambda P T^{3}-\frac{1}{P}(H T), \quad p_{c}=P .
\end{aligned}
$$

$P$ and $Q$ are a canonically conjugate pair of observables (they commute with the Hamiltonian constraint of these models), $T$ plays the role of an intrinsic time, and $H$ is essentially the Hamiltonian constraint, which now reads $H=k[11]$. In the associated $(P, H)$ representation, in which $\hat{Q}=i \partial_{P}$ and $\hat{T}=-i \partial_{H}$, the physical states of the quantum theory are provided by wavefunctions (distributions) of the form

$$
\Psi(P, H)=f(P) \delta(H-k)
$$

The reality conditions that correspond to Lorentzian gravity fix then the unique inner product [11]

$$
<\Phi, \Psi>=\int_{\mathbb{R}} d P \bar{h}(P) f(P),
$$

where $\Phi(P, H)=h(P) \delta(H-k)$ and denotes complex conjugation. As a consequence, the Hilbert space of quantum states turns out to be simply the space of functions $f(P) \in L^{2}(\mathbb{R})$.

In the adopted $(P, H)$ representation, and according to Ref. [11], the action of the operators $\left(\hat{b}, \hat{p}_{b}, \hat{c}, \hat{p}_{c}\right)$ is given by the direct translation of eqs. $(19,20)$ into operator language, with the only caveat that the factor $(H T)$ that appears in the definition

\footnotetext{
$\dagger$ The first equation in (20) corrects a missprint in eq. (51.c) of Ref. [11].
} 
of $c$ must be taken as a symmetric product. With this prescription (and neglecting again algebraic factors that never vanish when $c$ and $b$ are different from zero), the quantum equations (17) and (18) that characterize the Chern-Simons solution can be rewritten, respectively, as

$$
\begin{gathered}
{\left[(k-\hat{H})+(\hat{P} \hat{T})^{-1}\left(-i \hat{P}+\hat{Q} \hat{P}^{2}\right)\right] \Psi_{C S}(P, H)=0} \\
\left(-i \hat{P}+\hat{Q} \hat{P}^{2}\right) \Psi_{C S}(P, H)=0 .
\end{gathered}
$$

Notice that eqs. $(23,24)$ imply, in particular, that the Chern-Simons solution satisfies the Hamiltonian constraint, $(\hat{H}-k) \Psi_{C S}=0$. On the other hand, the presence of the singular operator $(\hat{P} \hat{T})^{-1}$ in eq. (23) poses no difficulties provided that eq. (24) is satisfied exactly. A trivial computation leads then to the explicit form of the Chern-Simons wavefunction (up to a constant) in the $(P, H)$ representation

$$
\Psi_{C S}(P, H)=f_{C S}(P) \delta(H-k), \quad f_{C S}(P)=\frac{1}{P}
$$

This wavefunction is obviously not normalizable with respect to the inner product (22), for $f_{C S}(P)=1 / P$ is not square integrable over the real axis. We thus conclude that the exponential of the Chern-Simons action is a physical state which, at least in the minisuperspaces that we are analysing, does not belong to the Hilbert space of quantum Lorentzian gravity.

We had commented above that, in the $(b, c)$ representation, the Ashtekar Hamiltonian constraint (16) admits also as solutions (in the distributional sense) the Green functions of the Wheeler-DeWitt equation that follows from eq. (4). In Ref. [11] we proved that, for Lorentzian gravity, these Green functions can be obtained from the discussed $(P, H)$ representation by restricting the domain of the variable $P$ to be the real positive axis. With this restriction, the normalizable physical states are still of the form (21), but now $f(P)$ must belong to $L^{2}\left(\mathbb{R}^{+}\right)$. Hence, the Chern-Simons wavefunction (25) turns out to possess an infinite norm also in this case. 
In conclusion, we have proved that the exponential of the Chern-Simons action does not provide an acceptable quantum state in the Lorentzian theories obtained from the non-perturbative canonical quantization of the anisotropic models (1). There are only two reasons that can prevent us from extending this result to the Ashtekar formulation of full Lorentzian gravity. The first caveat refers to the particular quantization that has been used in the analysis of the minisuperspace models, quantization that was obtained by assuming that the variables $b$ and $c$ run over the real axis [11]. For generic real domains of the Ashtekar variables $x$ and $y$, however, the phase space coordinates $b$ and $c$ may be complex in general, because the relations $(5,9)$ between these two sets of functions are not linear. It might then happen that, for reality conditions that allow complex domains for the classical variables $b$ and $c$ (so that $(x, y)$, and hence the densitized triad (6), are real), there exists a different canonical quantization in which the Chern-Simons wavefunction turned out to be normalizable. The second and most important reason is the drastic reduction of degrees of freedom that leads from full General Relativity to the studied family of minisuperspaces. This reduction may have significant physical consequences. In particular, the displayed non-normalizability of the Chern-Simons solution might simply be an artifact of the minisuperspace approximation analysed in this work.

In spite of these open problems, our results clearly indicate that one should not expect the exponential of the Chern-Simons action to be a proper quantum state in full Lorentzian gravity. It therefore seems that we do not know yet any exact solution to the quantum gravitational constraints which, in the presence of a cosmological constant, can describe a truly physical state.

This work was supported by funds provided by the Spanish Ministry of Education and Science Grant No. EX92-06996911. 


\section{References}

[1] A. Ashtekar, Phys. Rev. Lett. 57 (1986) 2244; Phys. Rev. D 36 (1987) 1587.

[2] A. Ashtekar, Lectures on Non-Perturbative Canonical Gravity, eds. L. Z. Fang and R. Ruffini (World Scientific, Singapore,1991).

[3] P. A. M. Dirac, Lectures on Quantum Mechanics, Belfer Graduate School of Science Monograph Series No. 2 (Yeshiva University, New York, 1964).

[4] A. Rendall, Class. Quantum Grav. 10 (1993) 2261.

[5] B. Brügmann, R. Gambini and J. Pullin, Nucl. Phys. B 385 (1992) 587; Gen. Rel. Grav. 25 (1993) 1.

[6] H. Kodama, Phys. Rev. D 42 (1990) 2548.

[7] L. N. Chang and C. Soo, Phys. Rev. D 46 (1992) 4257.

[8] C. Rovelli and L. Smolin, Phys. Rev. Lett. 61 (1988) 1155; Nucl. Phys. B 331 (1990) 80.

[9] I. Bengtsson, Class. Quantum Grav. 5 (1988) L139; 7 (1990) 27;

H. Kodama, Prog. Theor. Phys. 80 (1988) 1024;

S. Koshti and N. Dadhich, Class. Quantum Grav. 6 (1989) L223;

L. Bombelli and R. J. Torrence, Class. Quantum Grav. 7 (1990) 1747;

H. A. Kastrup and T. Thiemann, Nucl. Phys. B 399 (1993) 211;

N. Manojlović and A. Miković, Class. Quantum Grav. 10 (1993) 559.

[10] A. Ashtekar, R. S. Tate and C. Uggla, Int. J. Mod. Phys. D 2 (1993) 15;

N. Manojlović and G. A. Mena Marugán, Phys. Rev. D 48 (1993) 3704.

[11] G. A. Mena Marugán, Penn State Univ. preprint CGPG93/9-3, gr-qc/9309024, Class. Quantum Grav., to appear. 
[12] J. J. Halliwell and J. Louko, Phys. Rev. D 42 (1990) 3997.

[13] R. M. Wald, General Relativity (University of Chicago Press, Chicago, IL, 1984).

[14] A. Ashtekar, J. D. Romano and R. S. Tate, Phys. Rev. D 40 (1989) 2572. 